Communications in Physics, Vol. 14, No. 2 (2004), pp. 105-110

\title{
INTERACTION MODEL OF THE INTERFACE NASICON WITH AQUEOUS SOLUTION
}

\author{
NGUYEN TUYET NGA AND VO THACH SON \\ Institute of Engineering Physics, Hanoi University of Technology \\ C. DESLOUIS \\ Laboratoire Interface et Systems Electrochimique \\ CNRS UPR15, Tour 22- 4 Place Jussieu, 75252 Paris Cedex 05, France
}

\begin{abstract}
Complex impedance spectroscopy (CIS) study was carried out using the 4electrodes cell arrangement with NASICON membrane. The physical model developed in this work was based on own CIS characteristic of the system NASICON/solution, and it accurately described the experimental diagrams. The electronic parameters could be determined from the fittings with the designed model.
\end{abstract}

\section{INTRODUCTION}

Impedance spectroscopy is a very useful technique for evaluating the electronic characterization of different materials and processes. Giving an accurate model is very important for the evaluation of impedance spectra of interested materials. Nevertheless, it is not easy, for several classes of materials the suitable models are not even known yet. An example is that for the polycrystalline mixed conductors with semi-blocking internal and external boundaries (e.g. ceria, strontium titanate, mixed conducting cathode for Li-batteries, etc.) [1].

A popular tool for modeling the impedance spectra is equivalent circuits. A well known one is Randles circuit [2], which was derived for a simple electrode reaction in liquid electrochemistry. The presence of more complicated electrochemical systems leads to different modifications of Randles circuit. Often, the circuit is enriched intuitively by connecting additional resistors, capacitors and diffusion elements. Equivalent circuits also refer to polycrystalline materials. In such cases, not only electrodes but also grain boundaries mark the impedance spectra. If a pure ionic conductor is considered (e.g. YSZ) the presence of blocking grain boundaries can be described well by Bauerle's circuit [3] (two $\mathrm{RC}$ terms in series). If the fit of the measured data is not sufficiently good, the capacitors are replaced by constant phase elements, and diffusion elements with powers different from 0.5 are introduced. The criterion for the choice of a proper circuit is the quality of fit. But, as well known, several different circuits may fit the measured data with similar accuracy. So depending on every real system of material, there will be a suitable equivalent circuit.

In this paper, we will focus on the modeling of the impedance spectroscopy of NASICON ionic conductive material contacting aqueous solution. 


\section{EXPERIMENTAL}

\section{II.1 Materials}

Experimental details of the procedure for the NASICON sample elaboration have been described previously [4]. The NASICON of composition $\mathrm{Na}_{3} \mathrm{Zr}_{2} \mathrm{Si}_{2} \mathrm{PO}_{12}$ was synthesized by the sol-gel process. Ceramic pellets were obtained by pressing in disk shape with about $7 \mathrm{~mm}$ of diameter and $1.5 \mathrm{~mm}$ of thickness, sintering under air, at $1100{ }^{\circ} \mathrm{C}$ during 3 hours. Purity of the sample and crystallographic structure of material were checked by $\mathrm{X}$-ray diffraction (XRD). It is well known that in the as sintered NASICON may exist an excessive zirconia. In our case the NASICON containing a small amount of $\mathrm{ZrO}_{2}$ was observed. The conductivity was determined by an ac impedance spectroscopy. The samples used in the conductivity measurement have both two sides polished down to 1200 grit by emery paper.

\section{II.2. Four electrode impedance measurements}

The impedance data were obtained using Autolab. A four electrode configuration mode was used [4]. Silver electrodes were used as the reference electrodes. The membrane was bathed on both sides by the same solution so that the measurement cell was strictly symmetrical. Impedance measurements were performed in the frequency range $100 \mathrm{kHz}-$ $0.1 \mathrm{mHz}$, with an applied ac signal of $10 \mathrm{mV}$ at room temperature.

\section{RESULTS AND DISCUSSION}

\section{III.1. Impedance model}

The complex impedance diagrams of NASICON contacted $0.1 \mathrm{M} \mathrm{NaCl}$ aqueous solution after different times are presented in Fig. 1 and Fig. 2. They are of three loops.

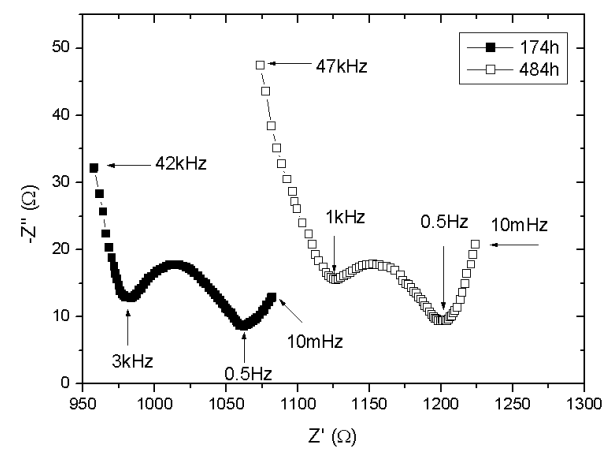

Fig. 1. Complex impedance diagram of NASICON contacted $0.1 \mathrm{M} \mathrm{NaCl}$ aqueous solution after $174 \mathrm{~h}$ and $484 \mathrm{~h}$

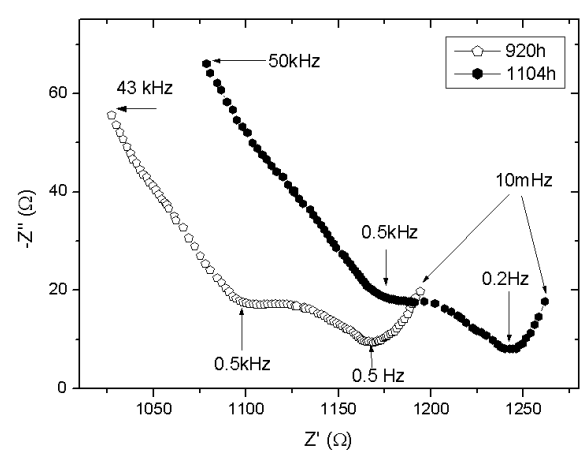

Fig. 2. Complex impedance diagram of NASICON contacted $0.1 \mathrm{M} \mathrm{NaCl}$ aqueous solution after $920 \mathrm{~h}$ and $1104 \mathrm{~h}$

The first loop at high frequency (HF) is related to bulk electric properties of the investigated material. The semicircle could characterized by a parallel $R-C$ circuit and 
the impedance is given by:

$$
Z=\frac{R}{1+(j \omega \tau)}
$$

with $\tau=R C$ - time constant.

However, as the material is not homogeniuos, HF loop does not display an ideal semi-circle shape (Fig.1, 2) but a quite flattened one. In this case, bulk resistance $R_{b}$ is replaced by constant phase element $C P E_{b}$, e.g., a Cole-Cole-type dispersion can be imposed as follows:

$$
Z_{H F}=\frac{R_{b}}{1+\left(j \omega \tau_{b}\right)^{\alpha_{b}}}
$$

where $\tau_{b}=R_{b} C_{b}, 0<\alpha_{b}<1$ with $C_{b}$ - bulk capacitance.

The equivalent circuit of the HF loop includes resistance $R_{b}$ in parallel with the $C P E_{b}$. The high frequency intersection of the semi-circle with the real axis corresponds to the total resistance of the NASICON membrance conducted to solution.

The second one at medium frequency $(M F)$ was contributed to the NASICON/solution interface process. It was not a perfect semi-circle and was flatter when ion-exchange time increased (Fig. 1,2). So $M F$ loop characterized by a parallel $R-C P E$ circuit and the imperdance $Z_{M F}$ is given by:

$$
Z_{M F}=\frac{R_{i}}{1+\left(j \omega \tau_{i}\right)^{\alpha_{i}}}
$$

where $R_{i}, C_{i}$ - interface resistance and capacitance with $0<\alpha_{i}<1$.

The third loop at low frequency (LF) is generally assigned to slow transport processes such as diffusion. The diffusion impedance in a medium of finite thickness without convection (finite Warburg impedance) is calculated as in Ref. [5]:

$$
Z_{L F}=Z_{W}=R_{d} \frac{t h\left(j \omega \tau_{w}\right)^{\alpha_{w}}}{\left(j \omega \tau_{w}\right)^{\alpha_{w}}}
$$

with $R_{d}$ - diffusion resistance, $\tau_{w}$ - time constant of impedance, and $0<\alpha_{w}<1$.

The complete impedance $Z$ is given by the sum of $Z_{H F}, Z_{M F}$ and $Z_{L F}$ in Eqs. $(2,3,4)$. The actually measured impedance also requires to take into account the solution resistance, $R_{S}$ :

$$
Z=R_{S}+Z_{H F}+Z_{M F}+Z_{L F}=R_{S}+\frac{R_{b}}{1+\left(j \omega \tau_{b}\right)^{\alpha_{b}}}+\frac{R_{i}}{1+\left(j \omega \tau_{i}\right)^{\alpha_{I}}}+R_{d} \frac{t h\left(j \omega \tau_{w}\right)^{\alpha_{w}}}{\left(j \omega \tau_{w}\right)^{\alpha_{w}}}
$$

Thus, equivalent circuit of the investigated system was described as Fig. 3

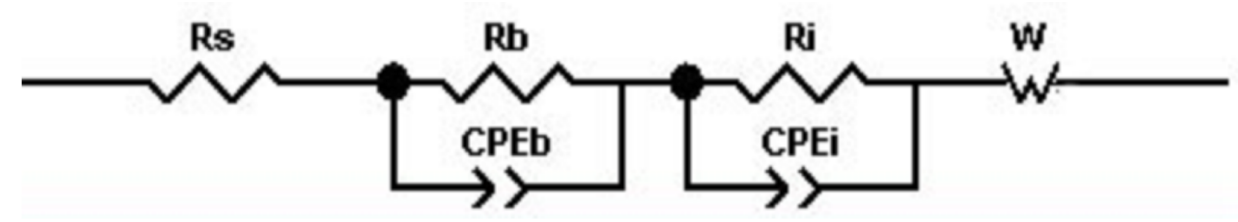

Fig. 3. The equivalent electrical circuit of the investigated system 


\section{III.2. Fitting results}

The different parameters of the equivalent electrical circuit were evaluated by means of a simplex fitting procedure [6]. The fitting results show an excellent agreement between the experimental and the theoretical diagrams as displayed in Fig. 4 and Fig. 5 in Nyquist (imaginary vs. real component).

Almost all the parameters in Eq. 5 were estimated from fitting, some of them have been determined directly from the measured impedance values. Resistance $R_{s}$ is unchange and estimted about $234 \Omega$.

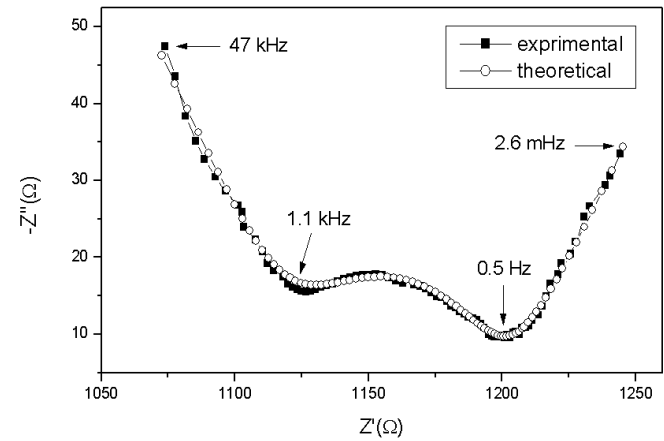

Fig. 4. Experimental and theoretical impedance diagrams after immersion during $484 \mathrm{~h}$

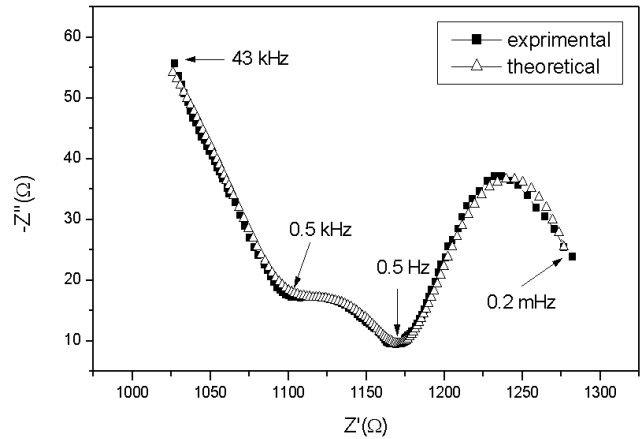

Fig. 5. Experimental and theoretical impedance diagrams after immersion during $920 \mathrm{~h}$

In Table 1, the values of the fitted parameters are reported. $\alpha_{b}, \alpha_{i}$ correspond to the CPE exponent of the HF, MF loops. $R_{b}, R_{i}$ and $R_{d}$ are resistances of the bulk, NASICON/solution interface and diffusion process respectively. $\tau_{b}$ and $\tau_{i}$ are time constants of the bulk, the NASICON/ solution interface and the diffusional process. $C_{b}, C_{i}$ were determined from equation $\tau=R C$.

Table 1. Value of the fitted parameters

\begin{tabular}{|l|l|l|l|l|l|l|l|l|l|}
\hline $\begin{array}{l}\text { Exposure } \\
\text { time }(\mathrm{h})\end{array}$ & $\tau_{b}$ & $\begin{array}{l}R_{b} \\
10^{-8} \mathrm{~s}\end{array}$ & $\begin{array}{l}\mathrm{C}_{b} \\
10^{-11} \mathrm{~F}\end{array}$ & $\alpha_{b}$ & $\begin{array}{l}\tau_{i} \\
10^{-3} \mathrm{~s}\end{array}$ & $\begin{array}{l}R_{i} \\
\Omega\end{array}$ & $\begin{array}{l}C_{i} \\
10^{-5} \mathrm{~F}\end{array}$ & $\alpha_{i}$ & $\begin{array}{l}R_{d} \\
\Omega\end{array}$ \\
\hline 174 & 5.14 & 736 & 6.98 & 0.71 & 1.94 & 188 & 1.0 & 0.45 & 96.6 \\
\hline 284 & 2.83 & 806 & 3.51 & 0.64 & 2.17 & 95 & 2.3 & 0.42 & 346 \\
\hline 335 & 2.34 & 856 & 2.73 & 0.51 & 3.27 & 79 & 4.1 & 0.49 & 99.4 \\
\hline 462 & 1.86 & 876 & 2.14 & 0.49 & 3.59 & 81 & 4.4 & 0.46 & 117 \\
\hline 484 & 2.09 & 886 & 2.35 & 0.50 & 3.50 & 83 & 4.2 & 0.45 & 255 \\
\hline 575 & 7.26 & 946 & 7.67 & 0.47 & 4.22 & 72 & 5.8 & 0.48 & 146 \\
\hline 790 & 1.96 & 866 & 2.26 & 0.43 & 3.96 & 72 & 5.5 & 0.46 & 402 \\
\hline 920 & 2.36 & 866 & 2.72 & 0.42 & 5.33 & 65 & 8.2 & 0.47 & 126 \\
\hline 1012 & 2.06 & 956 & 2.15 & 0.39 & 7.66 & 58 & 13.0 & 0.50 & 108 \\
\hline 1104 & 2.25 & 956 & 2.35 & 0.38 & 9.38 & 59 & 15.0 & 0.47 & 264 \\
\hline
\end{tabular}


The parameter values of Table 1 point out the following conclusions regarding some characteristics of the NASICON material.

The bulk resistance $R_{b}$ increased while the bulk capacitance decreased when ionexchange time increased. The increase of the bulk resistance could be ascribed to leaching of $\mathrm{Na}^{+}$ions from NASICON with resulting decrease in charge carrier concentration in electrolyte material as reported in Refs. $[7,8]$.

The CPE exponent $\alpha_{b}$ decreases which is suggested by grown-up of the pores of the material. By contrast, the interface resistance $R_{i}$ decreased and the interface capacitance increased. It could be related to the collection of $\mathrm{Na}^{+}$ions at NASICON/solution interface as proposed in Ref [4]. The CPE exponent $\alpha_{i}$, displays slight evolutions for each sample but is not far from 0.5 (ideal case) which is reasonable for a randomly dispersed micro porous matrix as shown by SEM picture (Fig. 6).

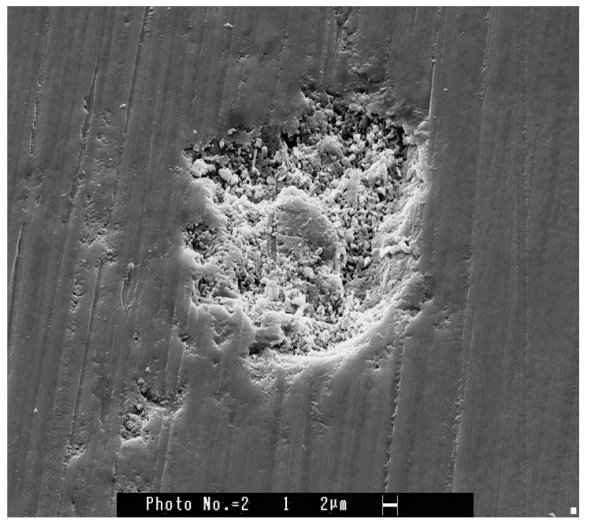

a)

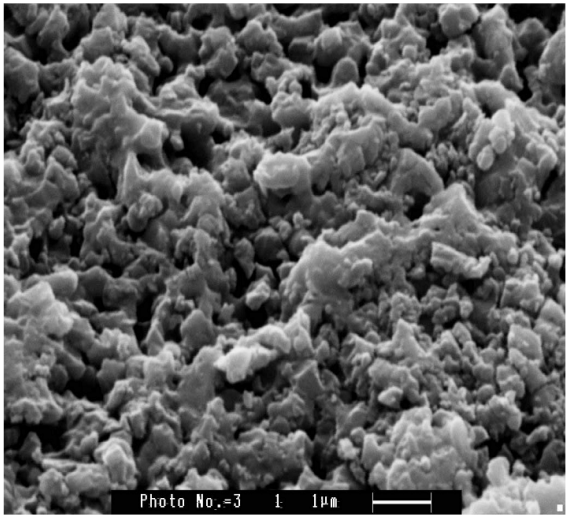

b)

Fig. 6. SEM micrograph of NASICON. Global view (a) and magnification image (b)

The values of $R_{d}$ proved for the diffusion process which was characterized by the third loop at very low frequency of impedance spectroscopy.

\section{CONCLUSIONS}

This work was intended to design a technique allowing access to some physical parameters which characterize the NASICON material contacted sodium cloride solution.

The proposed technique consisted in analyzing impedance spectroscopy of the material, in the so-called 4-electrodes cell arrangement which is currently used for investigating the transport properties of organic membranes. In comparison with an impedance model proposed here, the experimental data has provided changes of the NASICON electronic properties such as resistance, capacitance, etc... when the membrane was immersed in solution for a long period of time. 


\section{ACKNOWLEDGEMENTS}

The authors would like to thank Dr. C. Deslouis, F. Haubert and colleagues from the LISE, UPR 15 CNRS, France for their helpful assistance in carrying out this work.

\section{REFERENCES}

1. J. Jamnik, Solid State Ionics,157 (2003) 19

2. J.E.B. Randles, Discuss. Faraday Soc., 1 (1947) 11.

3. E. Bauerle, J. Phys. Chem. Solids, 30 (1969) 2657.

4. Nguyen Tuyet Nga, Vo Thach Son, Communication in Physics, 13 (2003) 252

5. J. B. Memet, P. Girault, R. Sabot, C. Compere, C. Deslouis, Electrochimica Acta, 47 (2002) 1043

6. J.A. Nelder, R. Nead, Comput. J., 7 (1965) 308.

7. Nguyen Tuyet Nga, Vo Thach Son, Tran Kim Lan, Phan Quoc Pho and Tran Viet Luc, Proceeding of the fifth Vietnamese - German Seminar on Physics and Engineering, Hue, 25 February - 02 March, 2002, pp. 33

8. R.O. Fuents, F. Figueiredo, F. M. Marques, J. I. Franco, Solid State Ionics, 139 (2001) 309 